\title{
Temperature Dependence of Photoluminescence from Epitaxial InGaAs/GaAs Quantum Dots with High Lateral Aspect Ratio
}

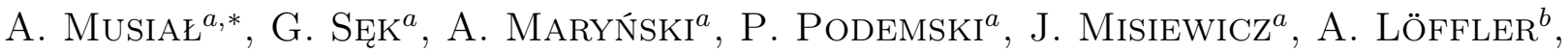 \\ S. Höfling ${ }^{b}$, S. Reitzenstein ${ }^{b}$, J.P. Reithmaier ${ }^{b, c}$ AND A. ForChel ${ }^{b}$ \\ ${ }^{a}$ Institute of Physics, Wrocław University of Technology, Wybrzeże Wyspiańskiego 27, 50-370 Wrocław, Poland \\ ${ }^{b}$ Technische Physik, University of Würzburg and Wilhelm-Conrad-Röntgen-Research Center for Complex \\ Material Systems (RCCM), Am Hubland, D-97074 Würzburg, Germany \\ ${ }^{c}$ currently at: Institute of Nanostructure Technologies and Analytics, Technische Physik \\ Universität Kassel, Germany
}

\begin{abstract}
Hereby, we present a study of a thermal quenching of emission from self-assembled epitaxial highly asymmetric quantum dots in InGaAs/GaAs material system for both ensemble and single dot regime. Pronounced interplay between the intensity of wetting layer and quantum dots originated emission was observed as the temperature was increased, evidencing a thermally activated energy transfer between the two parts of the system and an important role of the wetting layer in determining the optical properties of these anisotropic nanostructures. The carrier activation energies have been derived and possible carrier loss mechanisms have been analyzed. Single dot study revealed activation energies slightly varying from dot to dot due to size and shape distribution. The problem of the shape uniformity of individual quantum dot has also been addressed and possibility of additional carrier localization within the investigated structures has been found to be insignificant based on the recorded spectroscopic data.
\end{abstract}

PACS: 78.67.Hc, 65.80.-g

\section{Introduction}

Pronounced progress in nanostructures technology and development of new growth techniques result in a wide range of new quantum objects differing in geometry and details of quantum confinement with possibility of their engineering at the same time enabling to deal with new material systems. Those give opportunities to look for new configuration of materials and geometries most suitable for current optoelectronic applications, but also to create new conditions for testing the quantum electrodynamics phenomena in solid state. One of these relatively new groups of nanostructures are GaAs-based quantum dots elongated in one of the lateral dimensions $[1,2]$. They are very good examples of how a change in structure shape can have a strong influence on physical properties. Breaking the in-plane symmetry results in, e.g., selective polarization properties $[3,4]$ due to valence band states mixing $[5,6]$ and generally weaker quantum confinement $[7,8]$ as the growth conditions enable formation of larger nanostructures. Increased volume leads to an enhancement of the oscillator strength of optical transitions what makes these structures good candidates for achieving strong coupling regime in a quantum dot microcavity systems [2].

* corresponding author; e-mail: anna.musial@pwr.wroc.pl
Some of the characteristics of elongated GaAs-based QD ensemble are already known [9] and they have already been proven to have properties advantageous in quantum cryptography and computation applications [2], but the single dot regime needs to be further explored. Up to date, excitonic complexes and few-particle effects have been studied [8], but so far there has been no research considering the carrier loss and decoherence mechanisms in those structures on the single dot level. These can be essential for the evaluation of their application potential and the control of their properties being very important for the operation, especially the thermal stability, of future dot-based devices. The purpose of this report is to address the problem of thermal quenching of emission from elongated InGaAs quantum dots and to identify the primary carrier loss mechanisms basing on the activation energies determined from the temperature dependence of the photoluminescence intensity.

\section{Investigated structures and experimental details}

Investigated structures are InGaAs QDs grown epitaxially by MBE technique on GaAs substrate. The growth is driven by self-organization and results in quantum dots ensemble with certain size and shape distribution on InGaAs wetting layer (the Stranski-Krastanov growth mode). Low strain regime which is an effect of small 
lattice mismatch of approximately $2 \%$ between adjacent layers (quantum dots and substrate) and a low indium content (down to 30\%) enable growth of structures elongated in one of the lateral directions - [1-10] [1]. The nominal thickness of deposited InGaAs equals $4.5 \mathrm{~nm}$ (wetting layer and quantum dots) and causes relatively small QD surface density of about $5 \times 10^{9} \mathrm{~cm}^{-2}$ which is advantageous for single dot spectroscopy. Typical dimensions of investigated structures are as follows: $30-80 \mathrm{~nm}$ in length, 20-30 $\mathrm{nm}$ in width and 3-5 $\mathrm{nm}$ in height. The geometry is similar for all quantum dots with lateral aspect ratio between 2 and 4 .

Emission spectra of quantum dot ensemble (also polarization-resolved spectra) were measured in a standard photoluminescence setup where non-resonant excitation was provided by a semiconductor continuous wave laser $(660 \mathrm{~nm})$ and detection was based on a $0.5 \mathrm{~m}$ focal length monochromator with liquid nitrogen cooled InGaAs CCD linear detector. For single quantum dot (SQD) spectroscopy microscope objective with high numerical aperture (0.4) was used providing spatial resolution on the order of single $\mu \mathrm{m}$ (diffraction-limited size of the laser spot on the sample surface). The spectral resolution was kept below $100 \mu \mathrm{eV}$. To facilitate spectroscopy of single QDs mesas with sizes from $2 \mu \mathrm{m}$ down to $100 \mathrm{~nm}$ were fabricated on the sample surface (by combining electron beam lithography and reactive ion etching) limiting the number of excited structures to only few for the smallest mesa sizes.

\section{Experimental results and discussion}

The photoluminescence from quantum dot ensemble (measured on an unpatterned sample) revealed significant changes in relative intensities of the wetting layer (WL $-1.354 \mathrm{eV}$ at $5 \mathrm{~K}$ ) and quantum dots (QDs $1.320 \mathrm{eV}$ at $5 \mathrm{~K}$ ) emission (Fig. 1) when the temperature is raised from 5 to $60 \mathrm{~K}$. This feature is a fingerprint of thermally-induced energy transfer between the WL and QDs, and further carrier redistribution between the dots evidenced previously by time-resolved PL measurements [10].

At low temperature the WL emission dominates. The spectral separation between the WL and QDs emission is rather small and equals on average $35 \mathrm{meV}$ which shows that the confining potential for carriers in the investigated structures is quite shallow. The carrier transfer at low temperature is negligible and this can be traced back to the localized character of most of the wetting layer states with localization energy of approximately $2.5 \mathrm{meV}$ corresponding to a characteristic temperature of $30 \mathrm{~K}$ $[8,10]$. At elevated temperatures the carriers become extended in the WL plane enabling their transfer to the QDs which is manifested as a gradual increase in QD emission intensity in spite of its thermal quenching.

Another interesting feature is that the energy separation between WL and QDs emission is increasing with temperature. This effect can be explained in terms of

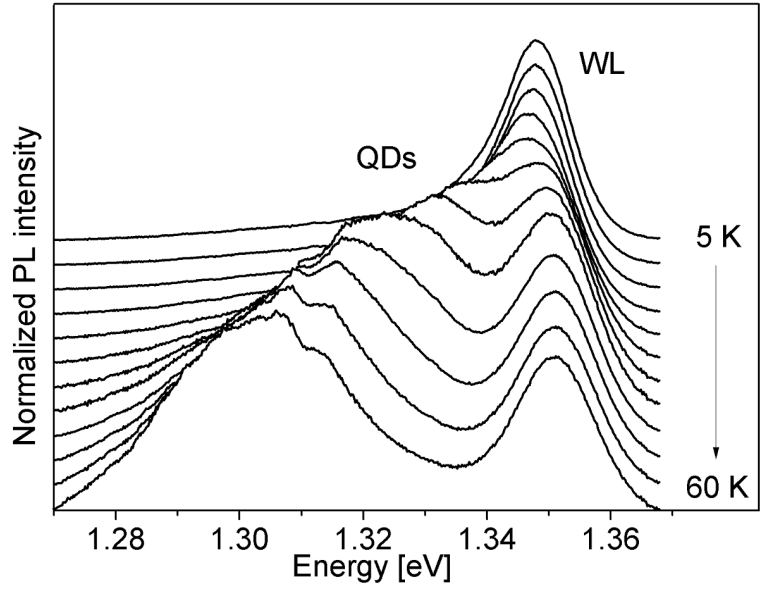

Fig. 1. Normalized photoluminescence spectra of InGaAs/GaAs QD ensemble in the temperature range from $5 \mathrm{~K}$ up to $60 \mathrm{~K}$.

carrier redistribution within the ensemble of QDs which has been activated after the carriers had been released from the traps in the WL and can be freely transferred to the dots. At the lowest temperatures the excited carriers once trapped by a random QD cannot be transferred into any other dot to find the absolute minimum of energy (the probability of such a process is very low) and are localized there until they recombine. This is due to the fact that the WL cannot mediate in the excitation transfer as most of its own states have a localized character.

This interpretation is further supported by the fact that no evidence of an interdot coupling has been observed for the investigated structures at low temperatures (because of low QD surface density, i.e. large interdot distances) and the excitation cannot be directly exchanged between the adjacent dots. The delocalization of the carriers from the WL states enables them to be transferred to the dots. Hence, the excitation redistribution within the QD ensemble starts leading to an increased occupation probability for the nanostructures with lower ground state energy (larger dots). This in consequence drives the increase of the energy separation between the QD and WL photoluminescence peaks.

Finally, as all the WL excitons become delocalized and the process of feeding QDs states saturates, the quenching mechanism becomes the only process driving the temperature evolution of the emission intensity. The activation energy obtained from the temperature dependence of the emission intensity amounts to approximately $30 \mathrm{meV}$ and corresponds well with the energy difference between WL and QDs emission at low temperature which points at the carrier release to the WL to be the primary carrier loss mechanism. This energy is slightly smaller than the spectral difference between the respective emission bands as a release of only one type of carrier is sufficient for the decay of the emission (especially that after the thermal 
activation of the carrier redistribution the energy separation is increased to almost $50 \mathrm{meV}$ ). Compared to the latter, the activation energy of a single carrier fits well to the approximate share of the total energy difference between the conduction and valence band.

Analogical measurements and analysis were performed in the SQD regime. Figure 2 presents the temperature series (in the $5-60 \mathrm{~K}$ temperature range) of $\mu \mathrm{PL}$ spectra from $1.5 \mu \mathrm{m}$ mesa. Despite a relatively large mesa size the low surface density enables one to obtain SQD emission spectra of a good quality with well-separated individual emission lines. In Fig. 3 exemplary Arrhenius plots for emission lines marked in Fig. 2 with red and green arrows, respectively, are presented. The quenching of individual emission lines can be usually well described by single activation energy. These energies vary from dot to dot between 15 and $30 \mathrm{meV}$ in agreement with results of measurements on the ensemble of QDs and reflecting QDs size (and as a consequence quantum confinement) distribution. This strongly indicates that the thermally activated release of carriers to the WL represents the main carrier loss mechanism. What is worth mentioning is that in case when the non-radiative recombination on the sidewall surface centers of the etched mesas can be neglected (i.e. for a dot in the middle of the mesa, or when the carrier transfer is limited), the WL has greater impact on the thermal quenching of the single dot emission than on QD ensemble. This is due to the fact that the probability of carrier once released from a QD to return to the same dot is negligible in comparison to the probability of returning to any of the QDs when the carrier is not lost and contributes to the ensemble emission anyway.

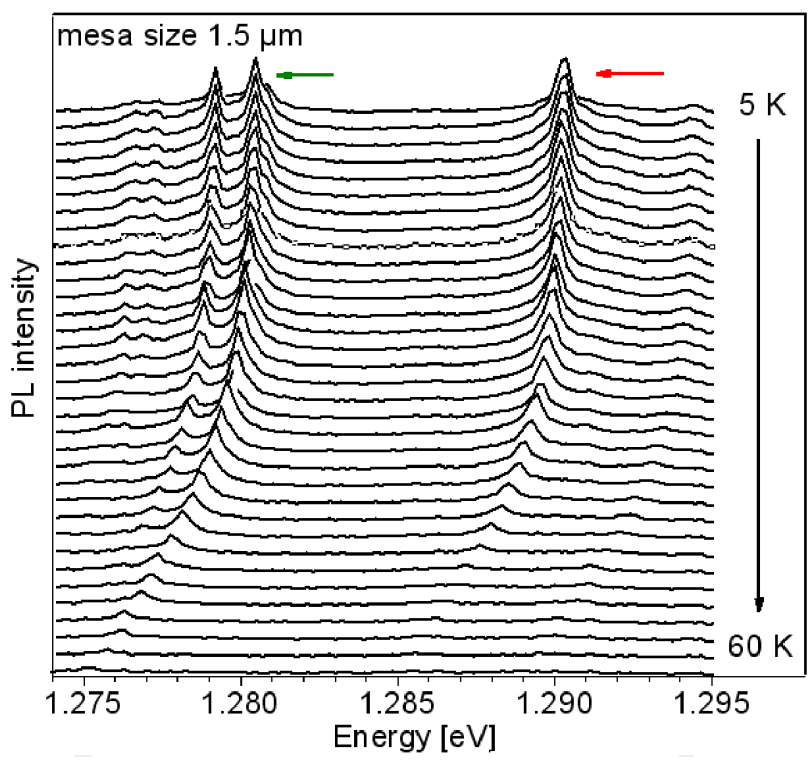

Fig. 2. Low energy part of microphotoluminescence spectra of InGaAs/GaAs single QDs in the temperature range from $5 \mathrm{~K}$ up to $60 \mathrm{~K}$.

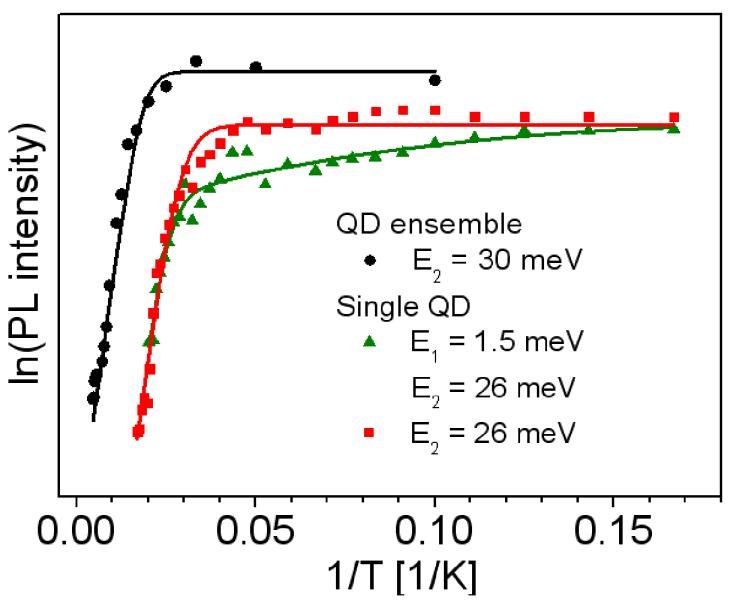

Fig. 3. Arrhenius plots (solid lines) for thermal quenching of QDs ensemble emission (black circles) and two exemplary single emission lines (green triangles and red squares) with activation energies obtained by the fitting procedure to experimental data (symbols). Results for QD ensemble are shifted vertically for clarity.

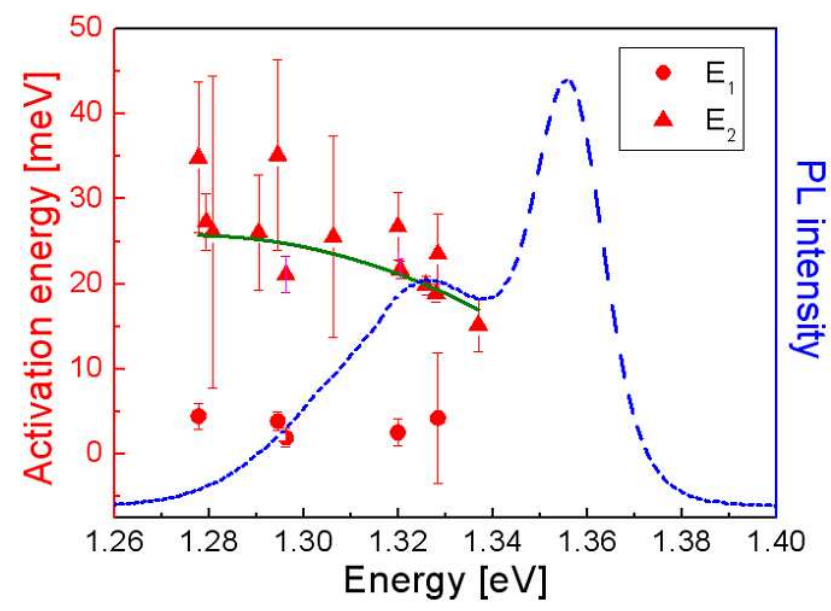

Fig. 4. Activation energy (red triangles $-E_{2}$ ) dependence on QD emission energy obtained from SQD experiments (green solid line denotes the respective tendency) shown against the photoluminescence spectra of the QD ensemble (blue dashed line). For some of the emission lines two activation energies can be distinguished (red circles $\left.-E_{1}\right)$.

It has been found that a few emission lines reveal second much smaller activation energy (green triangles in Fig. 3) of the order of single meV. At this point it should be clearly stated that the accuracy of our method is not better than $1 \mathrm{meV}$. This second activation energy cannot be observed on the QD ensemble level due to the carrier redistribution. It could be connected with a process of releasing carriers from a very shallow trapping potential, e.g., from potential fluctuations within the QD as it is possible for the indium content or shape to fluctuate on the scale of tens nm. This can happen due to relatively 
large lateral extension of the elongated QDs [12].

The occurrence of the second activation energy in very limited number of cases suggests that the QDs are generally uniform (which is also evidenced by a scanning electron microscopy (SEM) images of sample surface $[1,9]$ ) and the range of values for this energy (below $4 \mathrm{meV}$ ) shows that in the case of the existence of a trapping potential its localization energy is extremely low and can usually be neglected for determining the emission properties even at low temperatures.

Additionally, the fitting procedure to the Arrhenius plots [11] revealed that the contribution of this second quenching mechanism is three orders of magnitude lower than that of the main carrier loss mechanism, i.e. the efficiency of carrier release to the WL. This implies that the exciton is usually extended over the whole QD volume which corresponds well with previous observation of increased oscillator strength in these structures [2], i.e. in the weak confinement limit rather when the exciton Bohr radius is smaller than the lateral extension of the QD. Dispersion of the determined activation energies within the QD ensemble (Fig. 4) shows that the smaller energy is basically independent of the emission energy which is reasonable as it is not related to specific $\mathrm{QD}$ and is not its intrinsic property, but those potential fluctuations are rather randomly distributed over the sample. The second activation energy shows weak tendency to decrease with increasing emission energy which reflects the declined WL-QD energy separation for smaller dots (or lower indium content dots) as their energy states are pushed up because of stronger confining potential (or composition-related energy gap change).

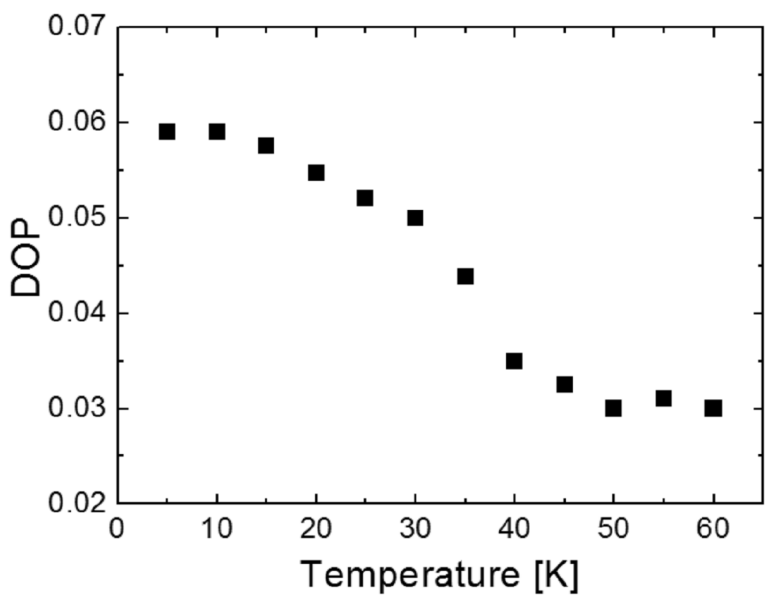

Fig. 5. The DOP of QD ensemble emission as a function of temperature.

The lack of any additional localization within the elongated dots was further tested in polarization-resolved photoluminescence measurements measurements on the QD ensemble. Low-temperature emission spectra in two orthogonal polarizations (along and transverse to the quantum dot elongation direction) were collected and the degree of linear polarization - DOP (using a common definition as a ratio between the difference and a sum of intensities of orthogonal polarizations taken at the maximum of emission band) of emitted radiation was determined on the level of few percent. This is unusually low for structures with so highly asymmetric in-plane geometry (lateral aspect ratio up to 4) [6, 12]. This apparent contradiction could be understood by taking into account a very shallow confining potential for both electrons and holes. The latter causes the carrier wave functions to leak significantly into the wetting layer, and thus effectively reduces the QD potential asymmetry. The temperature dependence of DOP can also be used as a probe of the inner localization in elongated nanostructures because of its sensitivity to the localizing potential symmetry. If present, the carrier trapping minima should be generally more isotropic and contribute to the emission significantly only at low temperatures (very small localization energy) decreasing the overall DOP [12], which has not been observed in the investigated case. In fact, for InGaAs/GaAs elongated QDs the DOP varies slightly with temperature decreasing within the whole temperature range (Fig. 5). This behavior is characteristic for uniform structures without potential fluctuations and reflects the increased contribution of higher energy states with increasing temperature (and higher states occupation) which are generally less polarized than the ground state [6].

\section{Conclusions}

The thermal quenching of photoluminescence on both ensemble and single dots was analyzed enabling the identification of primary carrier loss mechanism in InGaAs/ GaAs elongated QDs to be carrier release to the adjacent wetting layer with activation energy in the range of 15 $30 \mathrm{meV}$ depending on the energy separation between the specific QD and the WL. In a few cases single dot experiments revealed 2nd small activation energy which can be connected with releasing carriers from shallow potential fluctuations but efficiency of this process was found negligible. On this basis negligibility of additional trapping centers within the QDs was suggested and further confirmed in temperature dependence of the degree of linear polarization of emitted radiation, which corresponds well with previously proved increased oscillator strength of optical transitions, being a result of exciton extension over the whole QD volume providing larger coherence volume.

\section{Acknowledgments}

The authors acknowledge the financial support from the Polish Ministry of Science and Higher Education within grant No. N N202 181238 and the Foundation for Polish Science (FNP) and Deutsche Forschungsgemainschaft (DFG) - COPERNICUS Award. 


\section{References}

[1] A. Löffler, J.P. Reithmaier, A. Forchel, A. Sauerwald, D. Peskes, T. Kümmell, G. Bacher, J. Cryst. Growth 286, 6 (2006).

[2] J.P. Reithmaier, G. Sęk, A. Löffler, C. Hofmann, S. Kuhn, S. Reitzenstein, L. Keldysh, V. Kulakovskii, T. Reinecke, A. Forchel, Nature 432, 197 (2004).

[3] W. Sheng, Appl. Phys. Lett. 89, 173129 (2006).

[4] V. Zwiller, L. Jarlskog, M.E. Pistol, C. Pryor, P. Castrillo, W. Seifert, L. Samuelson, Phys. Rev. B 63, 233301 (2001).

[5] A.V. Koudinov, I.A. Akimov, Yu.G. Kusrayev, F. Henneberger, Phys. Rev. B 70, 241305(R) (2004).

[6] P. Kaczmarkiewicz, A. Musiał, G. Sęk, P. Podemski, P. Machnikowski, J. Misiewicz, Acta Phys. Pol. A 118, 333 (2010).

[7] M. Wimmer, S.V. Nair, J. Shumway, Phys. Rev. B 73, 165305 (2006)
[8] G. Sęk, A. Musiał, P. Podemski, M. Syperek, J. Misiewicz, A. Löffler, S. Höfling, L. Worschech, A. Forchel, J. Appl. Phys. 107, 096106 (2010).

[9] P. Poloczek, G. Sęk, J. Misiewicz, A. Löffler, J.P. Reithmaier, A. Forchel, J. Appl. Phys. 100, 013503 (2006).

[10] M. Syperek, A. Musiał, G. Sęk, P. Podemski, J. Misiewicz, A. Löffler, S. Höfling, L. Worschech, A. Forchel, in: 30th Int. Conf. on the Physics of Semiconductors - ICPS, Seul, 2010, AIP Conf. Proc., in press.

[11] G. Sęk, M. Motyka, K. Ryczko, F. Janiak, J. Misiewicz, S. Belahsene, G. Boissier, Y. Rouillard, Jpn. J. Appl. Phys. 49, 031202 (2010).

[12] A. Musiał, P. Kaczmarkiewicz, G. Sęk, P. Podemski, P. Machnikowski, J. Misiewicz, S. Hein, S. Höfling, A. Forchel, arXiv: 1107.4332v1 [cond-mat.mes-hall]. 